\title{
Reproducción de la morena, Gymnothorax equatorialis (Pisces: Muraenidae) en Jalisco y Colima, México
}

\author{
G. Lucano-Ramírez ${ }^{1}$, S. Ruiz-Ramírez ${ }^{1}$, J.A. Rojo-Vázquez ${ }^{1}$ \& G. González-Sansón ${ }^{2}$ \\ 1 Departamento de Estudios para el Desarrollo Sustentable de Zonas Costeras, Centro Universitario de la Costa Sur, \\ Universidad de Guadalajara, Gómez Farias 82, San Patricio-Melaque, C.P. 48980 Jalisco, México. Tel: (052-315) \\ 3556330, Fax (052-315) 3556331; lucanorg@costera.melaque.udg.mx; sruizram@costera.melaque.udg.mx; \\ jrojo@costera.melaque.udg.mx \\ 2 Centro de Investigaciones Marinas, Universidad de La Habana, Avenida Primavera 2808, Miramar, Ciudad de La \\ Habana, Cuba; gaspargonzalez@yahoo.com.es
}

Recibido 21-XI-2006. Corregido 07-V-2007. Aceptado 22-VIII-2007.

\begin{abstract}
Reproduction of the fish Gymnothorax equatorialis (Pisces: Muraenidae) in Jalisco and Colima, Mexico. A total of 707 Gymnothorax equatorialis were collected monthly in the Jalisco and Colima coast, Mexico, from December 1995 to December 1998 and from August to November 1999, in order to determine their reproduction patterns. Females outnumbered and had longer bodies (mean length $54.7 \mathrm{~cm})$ than males $(52.1$ $\mathrm{cm})$. The minimum, maximum and mean values of total fecundity were respectively 9660,99992 and 32029 eggs. The total body length at which 50 percent of individuals have ripe gonads $\left(L_{50}\right)$ was $43.7 \mathrm{~cm}$ for females and $42.7 \mathrm{~cm}$ TL for males. Ovaries had two main types of oocytes: small inmature in cromatin nucleolus phase $(85.1 \mu \mathrm{m})$ and large mature in secundary vitellogenesis phase $(701.6 \mu \mathrm{m})$. High numbers of spermatozoa were observed in the seminal tubes of ripe testicles, which presented a lobular development. Based in gonad features and temporal changes in the gonad-somatic index, it is concluded that G. equatorialis has two relatively short reproduction periods (at the middle and at the end of the year). Rev. Biol. Trop. 56 (1): 153-163. Epub 2008 March 31.
\end{abstract}

Key words: Gymnothorax equatorialis, Reproduction, spawning seasons, first maturity, fecundity, Coast of Jalisco and Colima.

La familia Muraenidae consta de 15 géneros y cerca de 200 especies. Se caracterizan por presentar una boca grande y forma corporal alargada, muscular y comprimida. Las especies de esta familia se distribuyen en mares templados y tropicales (Allen y Robertson 1994) y suelen ser capturadas como parte de la fauna acompañante en la pesquería de arrastre de camarón.

En la región de estudio (costa de Jalisco y Colima, México) se han registrado cuatro especies de esta familia (Aguilar-Palomino et al. 1996, Ruiz-Ramírez et al. 1997, RojoVázquez et al. 2001), entre las que se encuentra Gymnothorax equatorialis (Hildenbrand 1946).

G. equatorialis presenta aletas dorsal y anal muy cortas que se pueden distinguir del cuerpo. Las aletas forman un borde cubierto por piel. Las mandíbulas pueden cerrarse completamente, los dientes son alargados y forman una sola fila en cada mandíbula. La coloración de la región ventral del cuerpo varía de pardo o arena-claro a blanquecina. En todo el cuerpo presenta manchas blancas, en la cabeza las manchas son pequeñas y abundantes y a medida que se distribuyen hacia la cola se agrandan y distribuyen. G. equatorialis se localiza desde el Golfo de California, México, hasta Perú. La longitud máxima registrada es de $100 \mathrm{~cm}$. Es una especie bentónica que vive entre rocas y sobre fondos de arena y fango hasta 125 m de profundidad (Allen y Robertson 1994, McCosker y Rosenblatt 1995). 
Existe poca información disponible acerca de la biología y ecología de G. equatorialis. Los datos encontrados se refieren principalmente a la descripción y registro sistemático de la especie (Allen y Robertson 1994, McCosker y Rosenblatt 1995, Aguilar-Palomino et al. 1996, Ruiz-Ramírez et al. 1997) y a la comparación con nuevas especies (Lavenberg 1992, Hatooka y Randall 1992, Bussing 2000). El presente estudio tiene como objetivo analizar algunos aspectos reproductivos de G. equatorialis en las costas de Jalisco y Colima, México.

\section{MATERIALES Y MÉTODOS}

Área de estudio: la zona de estudio incluye desde Punta Farallón en el estado de Jalisco $\left(19^{\circ} 2^{\prime} \mathrm{N}, 105^{\circ} 01^{\prime} \mathrm{W}\right)$ hasta Cuyutlán en el estado de Colima $\left(18^{\circ} 55^{\prime} \mathrm{N}, 104^{\circ} 07^{\prime} \mathrm{W}\right)$. La línea de la costa es irregular y presenta tres accidentes importantes, constituidos por Bahía de Tenacatita, Bahía de Navidad y el grupo de bahías Manzanillo-Santiago, el resto de la línea costera está formada por zonas arenosas relativamente extensas y poco protegidas en donde se localizan las playas Cuitzmala, El Coco, Tepalcates y Cuyutlán. (González-Sansón et al. 1997).

Recolecta de muestras: Los ejemplares capturados se obtuvieron durante diciembre de 1995 a diciembre de 1998 y de agosto a noviembre de 1999, mediante muestreos nocturnos utilizando redes de arrastre a bordo del Barco de Investigación Pesquera BIP V propiedad de la Universidad de Guadalajara. El material obtenido se conservó en hielo hasta su procesamiento en el laboratorio. Los ejemplares se identificaron siguiendo la descripción de Allen y Robertson (1994). Cada individuo fue medido (LT) $( \pm 0.1 \mathrm{~cm})$ y pesado $(\mathrm{PT})$, además se registró el peso $( \pm 0.1 \mathrm{~g})$ de los organismos sin gónadas (Psg). Los ejemplares se disectaron para extraer las gónadas y se obtuvo el peso de las mismas $(\mathrm{PG})( \pm 0.1 \mathrm{~g})$. Se registró el sexo y el grado de madurez (inmadura y madura). Las gónadas fueron etiquetadas y preservadas en formol neutro al $10 \%$ para su posterior procesamiento histológico.

Análisis: La fecundidad parcial (Fp) de $G$. equatorialis, se estimó a partir de 55 ovarios previamente pesados. A pesar de que varió la longitud de los ovarios, entre ellos no se encontró diferencia en cuanto a la madurez y la distribución de los ovocitos. Por ese motivo, se tomó una muestra de $0.85 \mathrm{~g}$ de uno de los ovarios y se realizó el conteo de los ovocitos maduros (NO) de acuerdo con lo señalado por Cruz-Romero et al. (1991). Para determinar la fecundidad parcial se utilizó la ecuación:

$$
\mathrm{Fp}=[(\mathrm{NOxPG}) / \mathrm{PM}] \quad(1)
$$

Se realizó un análisis de regresión lineal simple, considerando la fecundidad como variable dependiente y el peso total como variable independiente.

Se calculó el índice gonadosomático (IGS) mensualmente mediante la expresión propuesta por Rodríguez-Gutiérrez (1992):

$$
\mathrm{IGS}=(\mathrm{PG} / \mathrm{Psg}) \times 100
$$

Se determinó la proporción sexual para la muestra total, mensualmente y por clase de longitud de talla y las posibles diferencias se analizaron mediante la prueba chi cuadrada, $\mathrm{X}^{2}$.

Para el cálculo de la talla de madurez se examinaron 333 hembras entre 31.6 a $79.0 \mathrm{~cm}$ (LT) y 203 machos de 21.5 a $69.5 \mathrm{~cm}$ (LT). Se definieron como individuos sexualmente maduros aquellos que presentaron ovocitos grandes (hembras) y líquido espermático (machos). Para estimar la longitud total en donde el 50 $\%$ de los individuos se encontraban maduros sexualmente $\left(\mathrm{L}_{50}\right)$, se ajustó la ecuación logística a la fracción de peces maduros por intervalos de clase de $4 \mathrm{~cm}$, según la expresión

$$
\mathrm{P}_{\mathrm{LT}}=1 /\left[1+\mathrm{e}^{(\mathrm{aLT}+\mathrm{b})}\right]
$$

donde $\mathrm{P}_{\mathrm{LT}}$ es el porcentaje de individuos maduros en esa longitud total (LT) o en esa 
clase de longitud total, a y b son parámetros de la ecuación de regresión (Echeverria 1987).

Proceso histológico: El procesamiento histológico consistió en la deshidratación con alcohol etílico, inclusión en parafina, obtención de cortes de $6 \mu \mathrm{m}$ y tinción con hematoxilinaeosina. Las observaciones se realizaron con un microscopio de luz. Se tomó una muestra de la parte media de los ovarios para describir las estructuras microscópicas y el grado de desarrollo, éste último según las categorías consideradas por Yamamoto y Yamazaki (1961). En total se realizaron 760 mediciones de diámetros de los ovocitos en diferentes fases de desarrollo utilizando una cámara digital.

Para la descripción histológica de los testículos, se tomó una muestra de la parte media de la gónada. El tipo de desarrollo y grado de madurez de las células de los testículos fueron determinados de acuerdo a lo descrito por Lucano-Ramírez (1998).

\section{RESULTADOS}

Se recolectaron 707 ejemplares de $G$. equatorialis, de los cuales 681 se pudieron distinguir entre hembras y machos. La distribución de frecuencias de longitudes de machos y hembras se presentan en la Fig. 1, se observa que la mayor parte de los individuos presentan longitudes entre 44 y $68 \mathrm{~cm}(98 \%)$ y algunos cuantos $(2 \%)$ se aprecian en las longitudes menores de 44 y mayores de $68 \mathrm{~cm}$. La longitud promedio de las hembras $(54.7 \mathrm{~cm})$ fue significativamente mayor $\left(\mathrm{t}_{\alpha(2), 685}=5.616\right.$, $\mathrm{p}<0.001)$ que la de los machos $(52.1 \mathrm{~cm})$. En la mayoría de los meses muestreados (19 de 28), las hembras presentaron una longitud promedio mayor que los machos, oscilando dicha longitud entre 49.0 y $57.0 \mathrm{~cm}$ (Fig. 2).

La proporción de machos y hembras para la muestra total fue de 1:1.4. La prueba de bondad de ajuste $\mathrm{X}^{2}$ sugiere que dicha proporción difiere de la proporción esperada $1: 1$ $\left(\mathrm{X}^{2}=19.88, \mathrm{p}<0.005\right)$. A su vez, la proporción de sexos por mes (Cuadro 1) muestra que en la mayoría de los meses donde se presentaron ambos (15 de 23), la proporción de hembras fue mayor que el de machos y en sólo cuatro meses la proporción de machos fue mayor que la proporción de hembras. Del análisis de la proporción sexual por clases de talla (Cuadro 2) no hay diferencia en los primeros cinco intervalos, mientras que en los últimos cuatro la proporción de hembras fue estadísticamente mayor que la de machos. La proporción de sexos en las tres variantes de análisis (muestra total, la distribución temporal y por clase de talla) mostró que el número de hembras es mayor que el de machos.

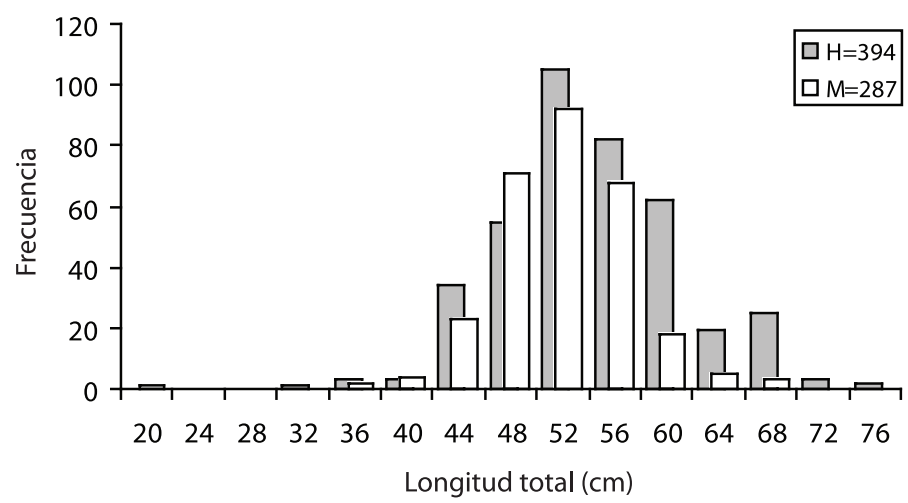

Fig. 1. Distribución de las frecuencias de tallas de hembras (H) y machos (M) de G. equatorialis en la costa de Jalisco y Colima, México.

Fig. 1. Distribution of the length frequency of females (H) and males (M) of G. equatorialis from the coast of Jalisco and Colima, Mexico. 


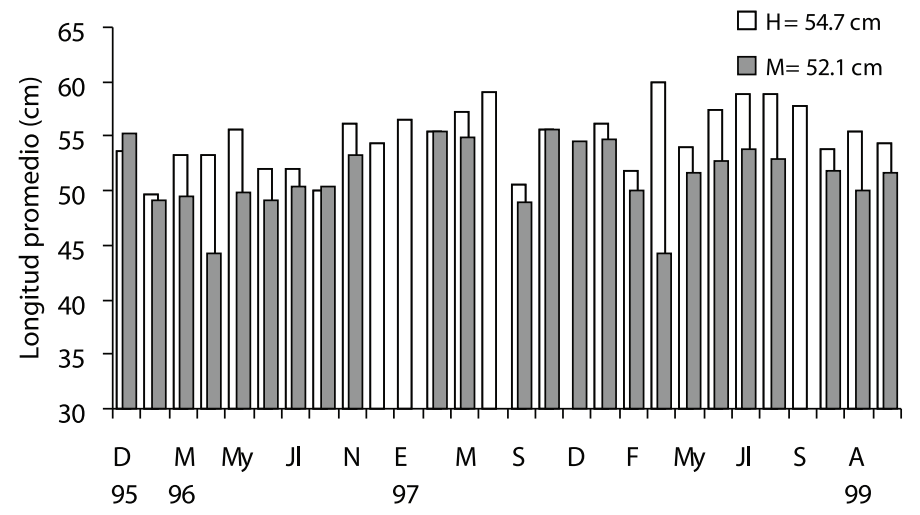

Fig. 2. Distribución mensual de las tallas promedio de hembras (H) y machos (M).

Fig. 2. Monthly distribution of the average length of females $(\mathrm{H})$ and males $(\mathrm{M})$.

\section{CUADRO 1}

Proporción sexual mensual de G. equatorialis

TABLE 1

Monthly sex ratio of $\mathrm{G}$. equatorialis

\begin{tabular}{|c|c|c|c|c|c|c|c|c|c|c|c|}
\hline & No. & $\mathrm{H}: \mathrm{M}$ & $X^{2}$ & DIF & $\mathrm{P}$ & & No. & $\mathrm{H}: \mathrm{M}$ & $X^{2}$ & DIF & $\mathrm{P}$ \\
\hline D 95 & 47 & 0.5 & 4.81 & $\mathrm{NO}$ & $>0.01$ & $\mathrm{O}$ & 27 & 0.9 & 0.09 & $\mathrm{NO}$ & $>0.75$ \\
\hline E 96 & 18 & 1 & 0.06 & $\mathrm{NO}$ & $>0.99$ & D & 2 & $2.0 *$ & 1.25 & $\mathrm{NO}$ & $>0.25$ \\
\hline M & 38 & 0.4 & 6.76 & SI & $<0.01$ & E 98 & 32 & 0.7 & 1.33 & $\mathrm{NO}$ & $>0.10$ \\
\hline A & 15 & 0.4 & 3.33 & $\mathrm{NO}$ & $>0.05$ & $\mathrm{~F}$ & 20 & 1.5 & 0.63 & $\mathrm{NO}$ & $>0.25$ \\
\hline My & 20 & 0.4 & 3.62 & $\mathrm{NO}$ & $>0.05$ & $\mathrm{M}$ & 15 & 0.9 & 0.17 & $\mathrm{NO}$ & $>0.50$ \\
\hline $\mathrm{J}$ & 19 & 0.5 & 2.97 & $\mathrm{NO}$ & $>0.05$ & My & 47 & 0.7 & 1.20 & $\mathrm{NO}$ & $>0.25$ \\
\hline $\mathrm{J} 1$ & 11 & 0.6 & 1.14 & $\mathrm{NO}$ & $>0.25$ & $\mathrm{~J}$ & 39 & 0.8 & 0.78 & $\mathrm{NO}$ & $>0.25$ \\
\hline $\mathrm{O}$ & 12 & 1.0 & 0.04 & $\mathrm{NO}$ & $>0.75$ & $\mathrm{Jl}$ & 5 & 1.5 & 0.10 & $\mathrm{NO}$ & $>0.75$ \\
\hline $\mathrm{N}$ & 36 & 0.6 & 2.01 & $\mathrm{NO}$ & $>0.10$ & A & 6 & 1.0 & 0.08 & $\mathrm{NO}$ & $>0.75$ \\
\hline D & 10 & $0.0^{*}$ & 11.05 & SI & $<0.01$ & $\mathrm{~S}$ & 7 & $0.0^{*}$ & 8.07 & SI & $<0.01$ \\
\hline E 97 & 26 & $0.0 *$ & 30.02 & SI & $<0.01$ & $\mathrm{D}$ & 72 & 0.8 & 1.01 & $\mathrm{NO}$ & $>0.25$ \\
\hline $\mathrm{F}$ & 15 & 0.9 & 0.17 & $\mathrm{NO}$ & $>0.50$ & A 99 & 8 & 0.3 & 2.56 & $\mathrm{NO}$ & $>0.10$ \\
\hline M & 39 & 1.2 & 0.17 & $\mathrm{NO}$ & $>0.50$ & $\mathrm{~N}$ & 101 & 1.5 & 3.39 & $\mathrm{NO}$ & $>0.05$ \\
\hline $\mathrm{Jl}$ & 2 & $0.0 *$ & 3.25 & $\mathrm{NO}$ & $>0.05$ & TOTAL & 691 & 0.7 & 90.31 & SI & $<0.01$ \\
\hline S & 2 & 1.0 & 0.25 & NO & $>0.50$ & & & & & & \\
\hline
\end{tabular}

No = número, $\mathrm{H}: \mathrm{M}=$ proporción de hembras y machos, $\mathrm{DIF}=$ diferencia, $\mathrm{P}=$ probabilidad.

No $=$ number, $\mathrm{H}: \mathrm{H}=$ females:males ratio, $\mathrm{DIF}=$ difference, $\mathrm{P}=$ probability. 
CUADRO 2

Proporción sexual por intervalo de longitud de

G. equatorialis

TABLE 2

Sex ratio of intervals of length of $\mathrm{G}$. equatorialis

$\begin{array}{lccccc}\text { Clase }(\mathrm{cm}) & \mathrm{N} & \% \text { de H } & X^{2} & \text { DIF } & \mathrm{P} \\ \text { menos de } 42 & 14 & 50.0 & 0.07 & \mathrm{NO} & >0.75 \\ 42.1-46.0 & 57 & 59.6 & 2.14 & \mathrm{NO} & >0.10 \\ 46.1-50.0 & 126 & 43.7 & 2.04 & \mathrm{NO} & >0.10 \\ 50.1-54.0 & 197 & 53.3 & 0.86 & \mathrm{NO} & >0.25 \\ 54.1-58.0 & 150 & 54.7 & 1.31 & \mathrm{NO} & >0.21 \\ 58.1-62.0 & 80 & 77.5 & 24.21 & \mathrm{SI} & <0.01 \\ 62.1-66.0 & 24 & 79.2 & 8.21 & \mathrm{SI} & <0.01 \\ 66.1-70.0 & 28 & 88.3 & 17.32 & \mathrm{SI} & <0.01 \\ \text { Mas de } 70.1 & 5 & 100 & 5.2 & \text { NO } & >0.01\end{array}$

No = número, $\%$ de $\mathrm{H}=$ porcentaje de hembras, DIF = diferencia, $\mathrm{P}=$ probabilidad.

No $=$ number, $\%$ of $\mathrm{H}=$ percentage of females, DIF $=$ difference, $\mathrm{P}=$ probability.

La distribución mensual del índice gonadosomático de cada sexo (Fig. 3) pone de manifiesto que en las hembras ocurren dos máximos de reproducción en el año, uno a mediados y el otro a finales del año. El IGS en machos, sin embargo, no manifestó una tendencia clara que permita definir la época de reproducción.
Al calcular la relación entre la longitud total y el porcentaje de organismos con gónadas maduras, en las hembras se encontró que el $50 \%$ de los individuos presentó gónadas maduras a la longitud de $43.7 \mathrm{~cm}$, mientras que en los machos fue a los $42.7 \mathrm{~cm}$ (Fig. 4 y 5 , respectivamente). La menor longitud que presentó un organismo fue de $36 \mathrm{~cm}$ en hembras y $38 \mathrm{~cm}$ en machos.

El menor número de óvulos contados ( 9 670) lo presentó una hembra de $57 \mathrm{~cm} \mathrm{y} \mathrm{el}$ mayor número de óvulos (99 992) correspondió a una hembra de $67 \mathrm{~cm}$, mientras que el promedio para todas las hembras fue de 32029 óvulos. El coeficiente de determinación $\left(\mathrm{R}^{2}\right)$ de la regresión lineal simple entre el número de huevos y el peso del organismo fue de 0.04 , lo que indica una baja relación entre las variables con el modelo aplicado.

Tanto los ovarios como los testículos presentaron dos características relevantes: primero que la longitud de estos órganos se extiende más atrás del poro genital y segundo, que la longitud de cada gónada de un individuo es diferente a la otra, presentando la gónada izquierda una reducción en su longitud entre el $30-40 \%$ con respecto a la derecha. Tanto en los ovarios como en los testículos la diferencia en longitud de cada una de las gónadas no afecta al desarrollo de los ovocitos o los espermatozoides, se puede encontrar el mismo grado de madurez y distribución de las células sexuales a lo largo de las gónadas.

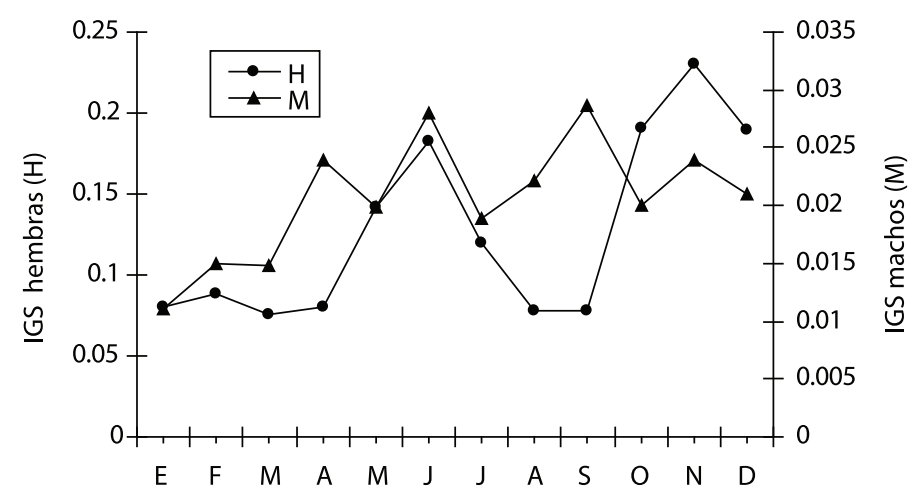

Fig. 3. Distribución mensual del índice gonadosomático (IGS) de hembras (H) y machos (M) de G. equatorialis.

Fig. 3. Monthly distribution of the Gonadosomatic index (IGS) of females (H) and males (M) of G. equatorialis. 


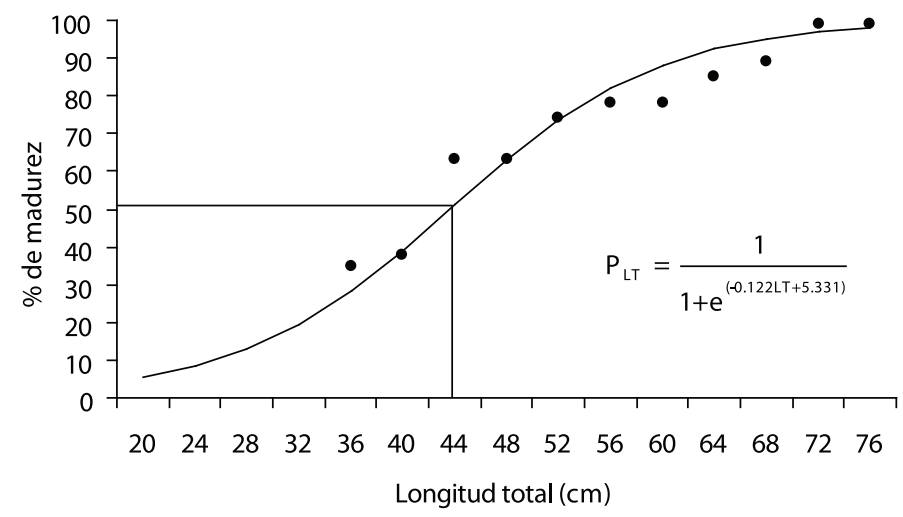

Fig. 4. Porcentaje de hembras maduras en las diferentes clases de talla.

Fig. 4. Percentage of mature females in the different length classes.

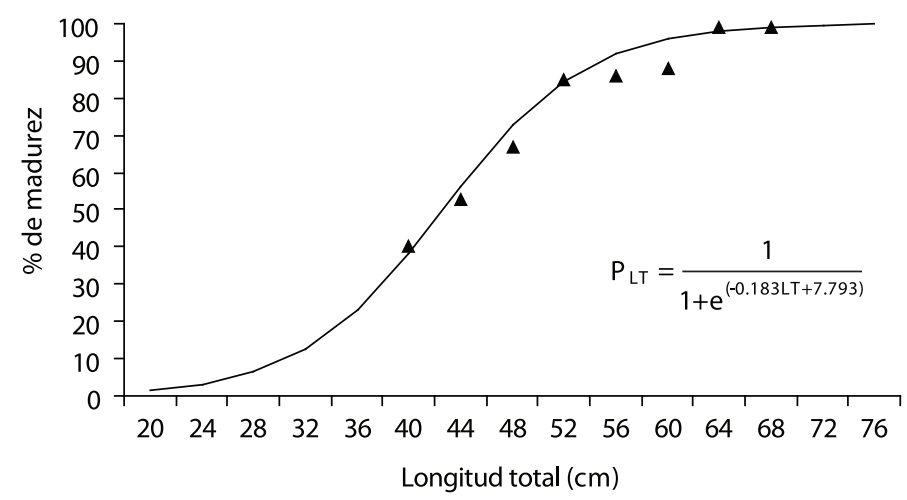

Fig. 5. Porcentaje de machos maduros en las diferentes clases de tallas.

Fig. 5. Percentage of mature males in the different length classes.

Las gónadas de G. equatorialis están adheridas por el mesorquio a la cavidad corporal, se extienden atrás de la cavidad branquial hasta unos centímetros después del poro anal. Los ovarios que presentan poco desarrollo, se asemejan a una cintilla por su longitud y por la evidente presión de la gónada; no se aprecia una túnica ovárica que envuelve a todos los ovocitos como ocurre en la mayoría de los peces. Los ovocitos se encuentran unidos entre sí mediante un epitelio, dando la apariencia de racimos. Los ovocitos con mayor grado de desarrollo son de tamaño relativamente grande, ya que se pueden observar a simple vista. El color de los ovarios inmaduros es amarillo y cuando los ovocitos están maduros presentan una coloración naranja, lo que caracteriza a un ovario maduro. El peso registrado de los ovarios inmaduros o mas pequeños fue de $1.6 \mathrm{~g}$ ( $0.6 \%$ del peso total de la hembra) y de los ovarios maduros o mas grandes fue de $201 \mathrm{~g}$ (44.2\% del peso total de la hembra).

A nivel microscópico se observaron cuatro etapas en el desarrollo de los ovocitos, las cuales se describen a continuación.

1. Ovocitos cromatina nucleolo $(\mathrm{CN})$ : Este tipo de ovocito pertenece a las primeras fases de desarrollo. Las células presentaron un diámetro medio de $85.1 \mu \mathrm{m}$ y 
representaron el $48.7 \%$ del total de ovocitos medidos (370 de 760 ovocitos medidos). Se caracterizan por presentar poco citoplasma y un núcleo grande, en cuyo interior se observan algunos nucleolos (Fig. 6A).

2. Ovocitos en perinucleolo: Se caracterizan por presentar en la periferia del núcleo algunos nucleolos y gran cantidad de citoplasma. Estos ovocitos presentaron un diámetro promedio de $129.8 \mu \mathrm{m}$ y constituyeron el $10.8 \%$ de la muestra total (Fig. 6B).

3. Ovocitos con vesículas vitelinas: Los nucleolos se observan en la periferia del núcleo y en el citoplasma se presentan vesículas vitelinas, que definen el inicio de la formación del vitelo o previtelogénesis. Presentaron un diámetro medio de 248.0 $\mu \mathrm{m}$ y un porcentaje de 4.9 sobre el número total de ovocitos analizados.

4. Ovocitos en vitelogénesis secundaria (VS): Estos se encuentran en las últimas fases de su desarrollo. Presentaron un diámetro promedio de $701.6 \mu \mathrm{m}$ y representaron el $35.6 \%$ del total de la muestra (271 de 760 ovocitos medidos). Se caracterizan por presentar un tamaño relativamente grande, $\mathrm{y}$ en el citoplasma se observa una gran cantidad de glóbulos de vitelo, los cuales darán origen al vitelo, esencial para la nutrición de la larva (6c).

Al igual que los ovarios, los testículos de G. equatorialis están adheridos a la cavidad corporal, se localizan por detrás de la cavidad branquial y se extienden hasta un punto posterior al poro anal. Los testículos son de forma aplanada y de color blanco, tanto los inmaduros como los maduros. Estos últimos, al momento de la extracción durante la manipulación, solían liberar líquido espermático blanquecino. El mayor peso de un testículo fue de $34 \mathrm{~g}$ (13.6 \% del peso total del macho) y el mínimo fue de $0.9 \mathrm{~g}(0.5 \%$ del peso del individuo $)$.

En el testículo, a nivel microscópico, se observó la túnica albugínea que rodea al testículo. El desarrollo de la línea germinal en el testículo es de tipo lobular. Los lóbulos están formados por numerosos cistos que se encuentran en diferentes estadios del proceso de espermatogénesis (espermatogonias, espermatocitos o espermatozoides). Sin embargo, dentro de cada cisto se pudo observar que las células se dividen de manera sincrónica, dando como resultado, que todas las células se encuentren en una misma etapa. Cuando las células que están dentro de los cistos se transforman en espermatozoides, estos son liberados en el lumen lobular, el cual esta en comunicación con el conducto espermático. La diferencia entre un testículo inmaduro (Fig. 7A y B) y uno maduro (Fig. 7C) es la cantidad de espermatozoides que se sitúan en el centro de los lóbulos o en el conducto espermático.

\section{DISCUSIÓN}

La proporción de sexos puede ser muy distinta según la especie, la población o la época del año (Nikolsky 1963) y puede experimentar cambios durante el periodo reproductivo y relación con las tallas (Franco 1992). En G. equatorialis no se conocen trabajos previos sobre esta especie o familia que aborden este aspecto. En la anguila (Anguilla rostrata), que pertenece al mismo orden de las morenas (Anguiliformes), Krueger y Oliveira (1997), señalan que el número de machos y hembras varía de acuerdo a un gradiente latitudinal, pero consideran que estas diferencias pueden ser resultado de métodos de muestreo inadecuados.

La longitud total máxima encontrada en $G$. equatorialis $(79.0 \mathrm{~cm})$ es mayor a la reportada por McCosker y Rosenblatt (1995), pero inferior a la señalada por Allen y Robertson (1994) (75 cm y $100 \mathrm{~cm}$ respectivamente). El área de muestreo de Allen y Robertson (1994) es muy amplia (Pacífico Centro-Oriental) en comparación con el área de muestreo del presente trabajo, quizá esta sea la causa por la cual la longitud máxima encontrada es mayor a la muestreada en este trabajo.

Krueger y Oliveira (1997), encontraron que en la Anguilla rostrata las hembras alcanzan tallas más grandes que los machos y en 

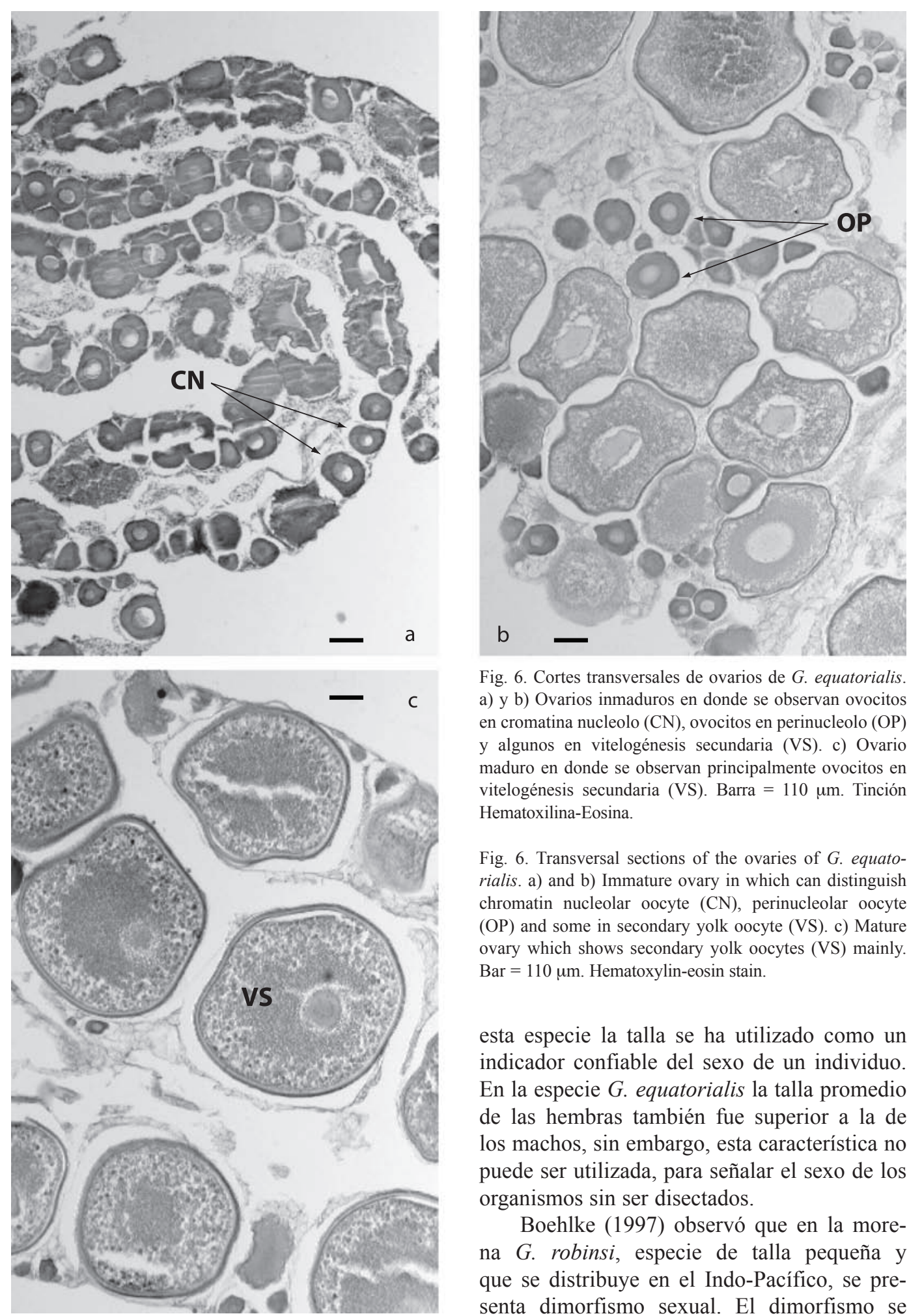

Fig. 6. Cortes transversales de ovarios de G. equatorialis. a) y b) Ovarios inmaduros en donde se observan ovocitos en cromatina nucleolo $(\mathrm{CN})$, ovocitos en perinucleolo (OP) y algunos en vitelogénesis secundaria (VS). c) Ovario maduro en donde se observan principalmente ovocitos en vitelogénesis secundaria (VS). Barra $=110 \mu \mathrm{m}$. Tinción Hematoxilina-Eosina.

Fig. 6. Transversal sections of the ovaries of G. equatorialis. a) and b) Immature ovary in which can distinguish chromatin nucleolar oocyte $(\mathrm{CN})$, perinucleolar oocyte (OP) and some in secondary yolk oocyte (VS). c) Mature ovary which shows secondary yolk oocytes (VS) mainly. Bar $=110 \mu \mathrm{m}$. Hematoxylin-eosin stain.

esta especie la talla se ha utilizado como un indicador confiable del sexo de un individuo. En la especie G. equatorialis la talla promedio de las hembras también fue superior a la de los machos, sin embargo, esta característica no puede ser utilizada, para señalar el sexo de los organismos sin ser disectados.

Boehlke (1997) observó que en la morena $G$. robinsi, especie de talla pequeña y que se distribuye en el Indo-Pacífico, se presenta dimorfismo sexual. El dimorfismo se 

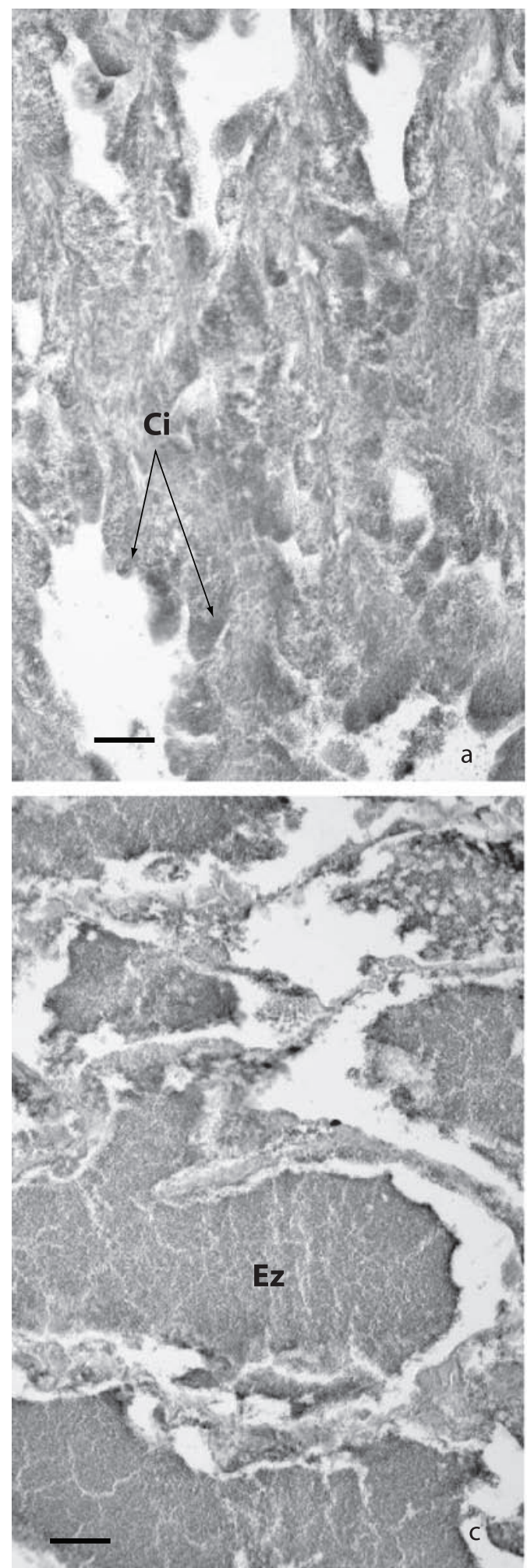

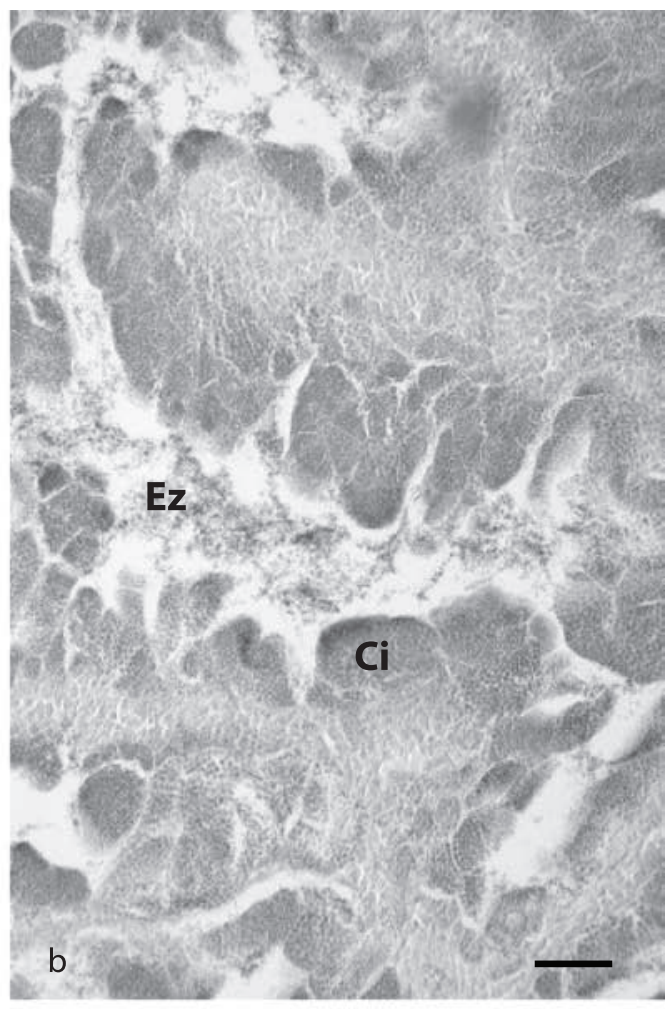

Fig. 7. Cortes transversales de testículos de G. equatorialis. a) y b) Testículos inmaduros los cuales están formados principalmente por cistos (Ci) y los espermatozoides (Ez) no se encuentran o son pocos los que se observan en el centro de los lóbulos. c) Testículo maduros en donde abundan los espermatozoides (Ez) en el centro de los lóbulos. Barra $=100 \mu \mathrm{m}$. Hematoxilina-Eosina.

Fig. 7. Transversal sections of the testes of $G$. equatorialis. a) and b) Immature testes which are formed by cysts (Ci), the sperm are absent or only few are present in the center of the lobules. c) Mature testes which show very abundant sperms (Ez) in the center of the lobules. Bar $=110 \mu \mathrm{m}$. Hematoxylin-eosin stain.

manifiesta en los dientes maxilares, que en las hembras son numerosos y biseriales, mientras que en los machos son pocos y uniseriales. En G. equatorialis no se presentaron características que apoyen el dimorfismo sexual externo de esta especie.

En el ovario de G. equatorialis se observaron principalmente dos clases de ovocitos, carácter distintivo de un ovario que presenta un desarrollo sincrónico por grupos. Una situación similar habita en la Anguila rostrata (Krueger y 
Oliveira 1997) donde se distinguen dos clases de ovocitos, unos pequeños y otros grandes. En los organismos que presentan este tipo de desarrollo sincrónico por grupos se observa que cuando se acerca la época de reproducción es común distinguir dentro del ovario dos tipos de ovocitos en desarrollo. En el primer grupo se observan ovocitos grandes que están en proceso de maduración terminal y un segundo grupo esta formado por ovocitos inmaduros, los cuales formarán el siguiente grupo de reclutamiento para el próximo evento o temporada de reproducción (Yamamoto y Yamazaki 1961). Los organismos que pertenecen a este grupo (sincrónico por grupos) por lo general se reproducen de una (en climas templados) a dos veces (en climas tropicales) al año y varias veces a lo largo de su vida, y se pueden caracterizar por presentar una época de desove corta y bien definida (Yamamoto y Yamazaki 1961).

Se considera que un gran número de los individuos incluidos en este estudio ya habían tenido por lo menos un evento reproductivo previo. Esto se infiere al hacer la comparación de las tallas promedio obtenidas en hembras $(54.7 \mathrm{~cm})$ y machos $(52.0 \mathrm{~cm})$, y que son superiores a la $\mathrm{L}_{50}$ de cada sexo.

G. equatorialis parece tener dos periodos reproductivos, uno a mediados y el otro a finales del año. Esta conclusión se apoya en el hecho de que las hembras presentan en los ovarios sólo dos grupos de ovocitos. Se asume que cada uno de estos grupos se corresponde con uno de los periodos reproductivos antes mencionados.

Como lo describen van Tienhoven (1983), Rodríguez-Gutiérrez (1992) y Jobling (1996), durante el proceso de espermatogénesis, las espermatogonias sufren numerosas divisiones mitóticas en el interior de los cistos. Cada lóbulo del testículo contiene numerosos cistos y dentro de cada cisto, las células germinales se dividen sincrónicamente, y así todas las células se encuentran en una misma fase de desarrollo. A su vez, dentro de los lóbulos, el proceso de espermatogénesis no es sincrónico, lo que significa que dentro de los lóbulos se pueden encontrar cistos en diferentes fases de desarrollo. Durante la espermatogénesis los cistos incrementan su tamaño y eventualmente se rompen para liberar los espermatozoides en el lumen lobular, el cual se continúa posteriormente con el conducto espermático, estas características y procesos mencionados por los autores fueron observadas en G. equatorialis.

Los resultados permiten inferir que la mayoría de los machos siempre se encuentran en actividad reproductiva o que cuando la fecundación se realiza por parejas, los machos emiten la cantidad de espermatozoides necesaria para fecundar a los huevos.

\section{AGRADECIMIENTOS}

A los investigadores, estudiantes del Departamento de Estudios para el Desarrollo Sustentable de Zonas Costera, así como a la tripulación del BIP V que participaron en los muestreos. Este trabajo se realizó bajo el permiso de Pesca de Fomento de la SEMARNAP No. 150995-214-03. La investigación fue financiada por la Universidad de Guadalajara y el CONACYT.

\section{RESUMEN}

Con el fin de analizar los aspectos reproductivos de Gymnothorax equatorialis se recolectaron mensualmente 707 organismos en las costas de Jalisco y Colima, México, de diciembre de 1995 a diciembre de 1998 y de agosto a diciembre de 1999. Las hembras fueron más numerosas y presentaron una longitud $(54.7 \mathrm{~cm})$ mayor a la de los machos $(52.1 \mathrm{~cm})$. La fecundidad total mínima fue de 9660 huevos, la máxima de 99992 y la media fue 32029 huevos. La talla en la que el $50 \%$ de los individuos presentan gónadas maduras $\left(\mathrm{L}_{50}\right)$ fue de $43.7 \mathrm{~cm}$ de longitud total en hembras y de $42.7 \mathrm{~cm}$ en machos. Los ovarios presentaron dos tipos de ovocitos, los pequeños inmaduros en fase cromatina nucleolo $(85.1 \mu \mathrm{m})$ y los grandes maduros en fase de vitelogénesis secundaria $(701.6 \mu \mathrm{m})$. En el testículo maduro se observó una gran cantidad de espermatozoides en el tubo seminal y el desarrollo del testículo es de tipo lobular. Con base en las características de las gónadas y la evolución temporal del índice gonadosomático, se concluye que $G$. equatorialis se reproduce dos veces al año (a mediados y finales del año). 
Palabras clave: Gymnothorax equatorialis, reproducción, temporada de reproducción, primera madurez, fecundidad, Costa de Jalisco y Colima.

\section{REFERENCIAS}

Aguilar-Palomino, B., J. Mariscal-Romero, G. GonzálezSansón \& L. Rodríguez-Ibarra. 1996. Ictiofauna demersal de fondos blandos de la plataforma continental de Jalisco y Colima, México, en la primavera de 1995. Cien. Mar. 22: 469-481.

Allen, G.R. \& D.R. Robertson. 1994. Fishes of the tropical eastern pacific. Univ. of Hawaii, Hawaii, EEUU.

Boehlke, E.B. 1997. Gymnothorax robinsi (Anguiliformes, Muraenidae), a new dwarf moray with sexually dimorphic distinction from the Indo-Pacific. Bull. Mar. Sci. 60: 648-655.

Bussing, W.A. 2000. Gymnothorax phalarus, a new eastern Pacific moray eel (Pisces: Muraenidae). Rev. Biol. Trop. 46: 439-446.

Cruz-Romero, M., E. Espino-Barr, J. Mimbela-López, A. García-Boa, L.F. Obregón-Alcaraz \& E. GirónBotello. 1991. Biología reproductiva en tres especies de Lutjanus en la Costa de Colima, México. Informe de Investigación, Colima, México.

Echeverria, T.W. 1987. Thirty-four species of California rockfishes: maturity and seasonality of reproduction. Fish. Bull. 85: 229-250.

Franco, L. 1992. Maduración sexual y fecundidad del carite (Scomberomosus maculatus) de las costas del estado Falcón, Venezuela. Zoot. Trop. 10: 157-169.

González-Sansón, G., B. Aguilar-Palomino, J. ArciniegaFlores, R. García de Quevedo-Machain, E. GodínezDomínguez, V. Landa-Jaime, J. Mariscal-Romero, J.E. Michel-Morfín \& M. Saucedo-Lozano. 1997. Variación espacial de la abundancia de la fauna de fondo blando en la plataforma continental de Jalisco y Colima, México (primavera 1995). Cien. Mar. 23: 93-110.

Hatooka, K. \& J.E. Randall. 1992. A new moray eel (Gymnothorax: Muraenidae) from Japan and Hawaii. Jap. J. Ichthyol. 39: 183-190.
Hildebrand, S.F. 1946. A descriptive catalog of the shore fishes of Peru. Bull. U.S. Nat. Mus. 189: 1-530.

Jobling, M. 1996. Enviromental biology of fishes. Chapman \& Hall, Fish and Fisheries Series, Londres, Inglaterra.

Krueger, W.H. \& K. Oliveira. 1997. Sex, size and gonad morphology of silver american eels Anguilla rostrata. Copeia 1997: 415-420.

Lavenberg, R.J. 1992. A new moray eel (Muraenidae: Gymnothorax) from oceanic islands of the South Pacific. Pac. Sci.. 46: 58-67.

Lucano-Ramírez, G. 1998. Ciclo reproductivo de Lutjanus peru (Nichols y Murphy 1922) (Pisces: Lutjanidae) en la costa sur de Jalisco. Tesis de maestría, UNAM, México D.F., México.

Mc Cosker, J.E. \& R.H. Rosenblatt. 1995. Muraenidae. In W. Fischer et al. (eds). Guía FAO para la identificación de especies para los fines de la pesca. Pacífico centro-oriental. FAO, Roma, Italia.

Nikolsky, G. 1963. The ecology of fishes. Academic, Londres, Inglaterra.

Rodríguez-Gutiérrez, M. 1992. Técnicas de evaluación cuantitativas de la madurez gonádica en peces. AGT, México D.F., México.

Rojo-Vázquez, J.A., B. Aguilar-Palomino, V. Galván-Piña, E. Godínez-Domínguez, S. Hernández-Vázquez, S. Ruiz-Ramírez \& G. Lucano-Ramírez. 2001. Ictiofauna de la pesquería ribereña en Bahía de Navidad, Jalisco, México, asociada al evento El Niño 1997-1998. Rev. Biol. Trop. 49: 915-929.

Ruiz-Ramírez, S., G. Lucano-Ramírez \& J. MariscalRomero. 1997. Length-Weight relationships of softbottom demersal fishes from Jalisco and Colima states, Mexico. Naga, ICLARM Q. 20: 62-63.

van Tienhoven, A. 1983. Reproductive physiology of vertebrate. Cornell, Nueva York, EEUU.

Yamamoto, K. \& F. Yamazaki. 1961. Rhythm of development in the oocyte of the gold-fish, Carassius auratus. Bull. Fac. Fish. Hokkaido Univ. 12: 93-114. 
